\title{
Face Image Denoising Method Based on Fourth-order Partial Differential Equations
}

\author{
Wengcang Zhao ${ }^{1,}$, Fan Wang ${ }^{2, b}$ \\ ${ }^{1,2}$ Qingdao University of Science and Technology,Zhengzhou Road,Qingdao,China

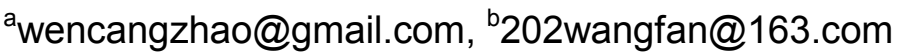

\begin{abstract}
Keywords: face image denoising; P-M non-linear diffusion; fourth-order partial differential equation(PDE)
\end{abstract}

\begin{abstract}
In order to improve the effect of face image denoising, this paper put forward several face image denoising methods based on partial differential equations, including P-M non-linear diffusion equations and fourth-order partial differential equations. We use those two methods by establishing non-linear diffusion equations and fourth-order anisotropic diffusion partial differential equation. The P-M non-linear diffusion denoising method can remove noise in intra-regions sufficiently but noise at edges can not be eliminated successfully and line-like structures can not be held very well. While the fourth-order partial differential equations denoising can retain the local detail characteristics of the original face image. Finally, through the experimental results we can see the effect of the fourth-order partial differential equations denoising is better, which makes the later face image processing more accurate and promotes the development of face image processing.
\end{abstract}

\section{Introduction}

Face recognition is one of the controversial research projects in recent years, it's relevant technologies play a very important role on the development of identity recognition, city security and other fields ${ }^{[1]}$. And the pre-denoising of face image is an important prerequisite to ensure the success in face recognition. But because face image is complex, and feature points are widely distributed, so in the early image denoising process, it is more difficult to ensure denoising effect while still keep the details of the characteristics of the face images.

\section{The Traditional P-M Nonlinear Diffusion Method}

Firstly this paper analyzes the traditional P-M non-linear diffusion method.The P-M equation is put forward by Perona and Malik in $1990^{[2]}$. It can be seen as an isotropic uniform thermal diffusion equation. We can make the "conduction coefficient" depend on local features of image in diffusion process for denoising while preserving the image edges. In other words, conduction coefficient can automatically increase at the relatively flat areas of image, which can ensure that the smaller irregular ups and downs (noise) in flat area is smooth.And near the edges of the image,conduction coefficient can be reduced automatically, so the image edges can be hardly affected ${ }^{[3]}$. Based on the above methods, we can get a better denoising effect. The non-linear diffusion model is shown in equation $(1)^{[4]}$

$$
\left\{\begin{array}{l}
\frac{\partial \mathrm{u}_{t}}{\partial t}=\operatorname{div}\left[g\left(\left|\nabla u_{t}\right|\right) \nabla u_{t}\right] \\
u(x, y, 0)=u_{0}(x, y)
\end{array}\right.
$$

Where $u_{t}$ denotes the image at time t. $\nabla u_{t}$ is the gradient of the image. $g\left(\nabla u_{t} \mid\right)$ is called diffusion function whose value indicates diffusion intensity, and usually $g\left(\left|\nabla u_{t}\right|\right)$ is a non-negative smooth monotone decreasing function. This method in image denoising can avoid deficiencies well about that thermal diffusion can not select denoising on image edges and smooth regions. However, the 
image denoising method based on the P-M equation also has its own drawbacks. This method is unable to get rid of the big noise points in image. In addition, if there is excessive denoising on image, it will lead to image and gray image into a subdivision, namely the staircasing effect.

It is inconvenience for follow-up face image processing. In order to compensate for its lack of face image denoising, this paper proposes a method of the fourth-order partial differential equations applied to the face image denoising.

\section{The Fourth-order Partial Differential Equations}

The P-M equation processed image will cause the staircasing effect, so in order to solve this problem, You Yu-Li and M.Kavch ${ }^{[5]}$ put forward the following fourth-order anisotropic diffusion partial differential equation in 2000.

$$
\left\{\begin{array}{l}
\frac{\partial u}{\partial t}=-\nabla^{2}\left[\mathrm{~g}\left(\nabla^{2} u\right) \nabla^{2} u\right] \\
u(x, y, 0)=u_{0}(x, y)
\end{array}\right.
$$

Equation (2) corresponding the energy functional

$$
E(u)=\int \mathrm{f}\left(\left|\nabla^{2} u\right|\right) d \Omega
$$

The corresponding Euler equation

$$
\nabla^{2}\left[\mathrm{~g}\left(\nabla^{2} u\right) \nabla^{2} u\right]=0
$$

Where $g(\cdot)$ is a positive and non-increasing function. The definition of $\mathrm{f}(\cdot)$ is: $g(\cdot)=f^{\prime}(s) / \mathrm{s}$. $\mathrm{f}\left(\left|\nabla^{2} u\right|\right)$ is a non-negative and non-decreasing function.In the above equations, $\mathrm{Yu}$-Li and M.Kaveh let $g(s)=\frac{1}{1+(s / k)^{2}}$, so $f(s)=\frac{k^{2}}{2} \ln \left(1+\frac{s^{2}}{k^{2}}\right)$, Where $\mathrm{k}$ is a constant.

The benefits of the face image denoising by equation (2) is that we can use the segmented cant (it is piecewise slash for the plane curve) to take the place of the outline of the face boundary approximatly, which can eliminate the staircasing effect of the face images, make the denoising face image clearer.

But the equation also have shortcomings, that is, filtering out the noise of the smooth region will cause the image not-flat at the same time.In addition it can also not remove the salt and pepper noises. Therefore we need to improve the above equations.

When we add noises to the face image, the area of the image will be increased.So we can use the volume of the image take the place of ${ }^{E(u)^{[6]}}$ in the equation (2), then ${ }^{[7]}$

$$
E(\mathrm{u})=\int d \Omega
$$

Where $d \Omega$ stands for the integral surface area.

For plane curve, equation (5) is equation (6)

$$
E(u)=\int \sqrt{1+u_{x}^{2}} d x
$$

For space plane, equation (5) is equation (7)

$$
E(u)=\int \sqrt{1+u_{x}^{2}+u_{y}^{2}} d x d y
$$

Using gradient descent method, we can get the solution of equation (6)

$$
u_{t}=K
$$


Using gradient descent method, we can get the solution of equation (7)

$$
u_{t}=H
$$

Here $\mathrm{K}$ represents the curvature of the plane curve.H represents space surface curvature of the average.

We can give the definition $f(s)=\sqrt{1+s^{2}}$ in the equation (7), where $s=\left|\nabla^{2} u\right|$. Combined with the thought of $\mathrm{Yu}-\mathrm{Li}$ and M.Kaveh,we can use the following equation to replace $\mathrm{f}($.$) in the equation(3)$

$$
f\left(\nabla^{2} u\right)=\sqrt{1+\left(\nabla^{2} u\right)^{2}}
$$

Then $\frac{\partial u}{\partial t}=-\nabla^{2}\left[\mathrm{~g}\left(\nabla^{2} u\right) \nabla^{2} u\right]$ in equation (7)can be improved,

$$
\begin{gathered}
\frac{\partial u}{\partial t}=-\nabla^{2}\left(\frac{1}{\sqrt{1+s^{2}}} \nabla^{2} u\right) \\
g(s)=\frac{1}{\sqrt{1+s^{2}}} \text { inequat }
\end{gathered}
$$

After the improvement, equivalently $\sqrt{1+s^{2}}$ inequation (3). So $f(s)=\sqrt{1+s^{2}}$ in energy function equation.

The improved fourth-order partial differential equations (11) can overcome the shortcomings that the $\mathrm{Yu}-\mathrm{Li}$ and $\mathrm{M}$.Kaveh equation can not remove the salt and pepper noises. The original image and processed images are shown in Figure 1.

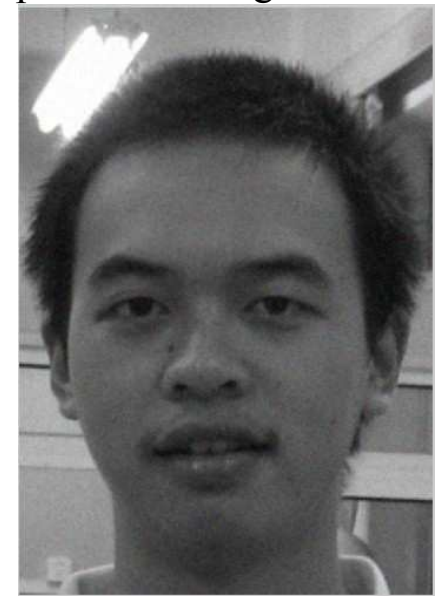

the original image

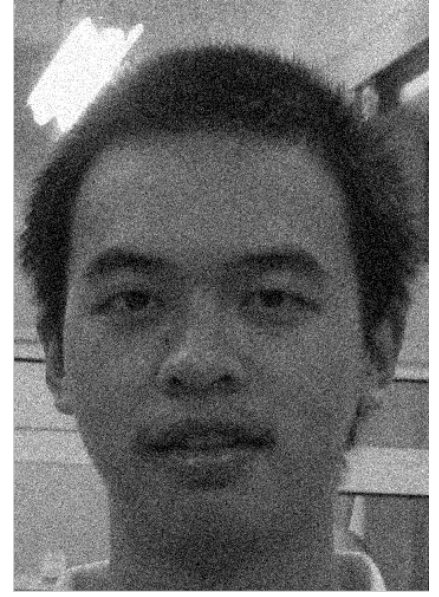

the adding noise image

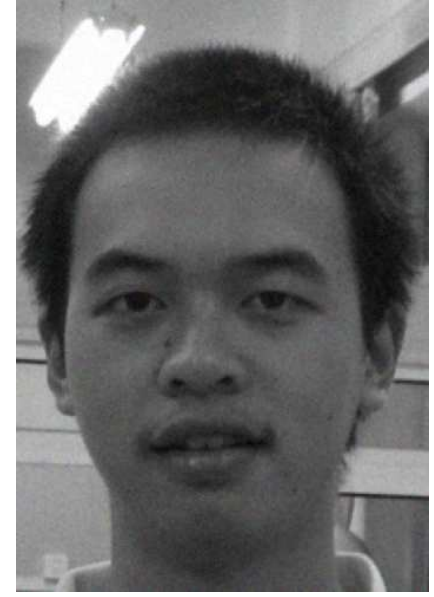

P-M nonlinear diffusion denoising

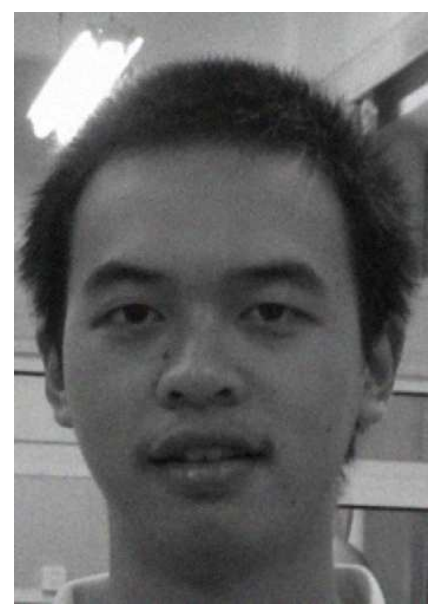

fourth-order PDE denoising

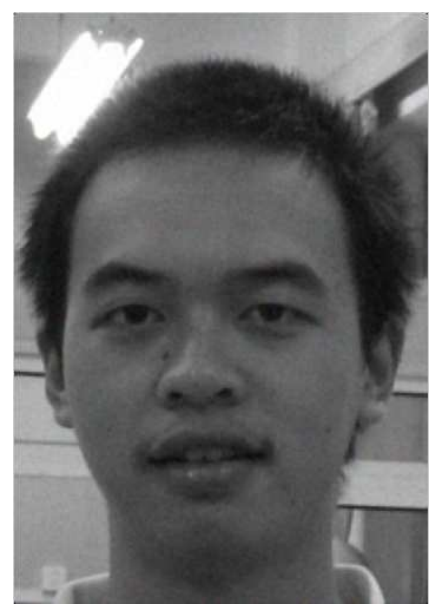

improved fourth-order PDE denoising

Fig.1.The original image and processed images 
Through comparing the P-M non-linear diffusion method and the fourth-order partial differential equations denoising, we can found the four-order partial differential equation denoising can make local details of face image clearer, the effec of noise filter more apparent, face image after filter clearer, which is more beneficial for post processing and feature extraction of the image.

\section{Summary}

This paper analyzes the traditional P-M non-linear diffusion method and fourth-order partial differential equation applied for denoising images. Finally, the renderings of the face image denoising are given.Through the analysis,staircasing effect is restrained and image edge blurring is avoid by the fourth-order partial differential equation denoising,which has greater local self-adaptability and higher flexibility. It is also more beneficial for the latter part of the face image processing and facial feature extraction.

\section{References}

[1] Chellappa M,Wilson C,Sirohey S,Human and machine recognition of faces:a survey, J. Proceeding of the IEEE. 83 (1995) 705-741.

[2] Perona P,Malik J, Scale-space and edge detection using anisotropic diffusion, J. IEEE Trans on Pattern Anal Machine Intell. 12 (1990) 629-639.

[3] Xinwu Liu, Lihong Huang, Zenyuan Guo, Adaptive fourth-order partial differential equation filter for image denoising, J. Applied Mathematics Letters. 24 (2011) 1282-1288.

[4] Linfang Wu,Naping Chen, Main models for image noise removal based on PDEs, J. Journal of Minjiang University. 27 (2006) 25-28.

[5] You Yu-Li,Kvech M, Four-order partial differential equations for noise removal, J. IEEE Trans on Image Processing. 9 (2000) 1723-1729.

[6] Kenneth R C, Digital image processing, Prentice Hall Internationl:Englewood Cliffs. 1988.

[7] Xiurui Geng,Suoping Li, Four-order partial differential equations used for image denoising,J. Gansu Industrial University Press. 28(2002) 256-259. 